\title{
Algorithms for Scheduling Real-Time Tasks with Input Error and End-to-End Deadlines
}

\author{
Wu-chun Feng, Member, IEEE, and Jane W.-S. Liu, Fellow, IEEE
}

\begin{abstract}
This paper describes algorithms for scheduling preemptive, imprecise, composite tasks in real-time. Each composite task consists of a chain of component tasks, and each component task is made up of a mandatory part and an optional part. Whenever a component task uses imprecise input, the processing times of its mandatory and optional parts may become larger. The composite tasks are scheduled by a two-level scheduler. At the high level, the composite tasks are scheduled preemptively on one processor, according to an existing algorithm for scheduling simple imprecise tasks. The low-level scheduler then distributes the time budgeted for each composite task across its component tasks so as to minimize the output error of the composite task
\end{abstract}

Index Terms-Real-time systems and applications, scheduling, imprecise computation, error, end-to-end timing constraints.

\section{INTRODUCTION}

A HARD real-time system contains tasks which must produce logically correct results within certain timing constraints. In a system where the processing times of tasks vary, transient overloads may be unavoidable. A hard realtime system must remain robust and maintain an acceptable level of performance under a transient overload. The imprecise-computation technique [1], [2], [3], [4], [5] was introduced as a way to deal with transient overloads. The technique is motivated by the fact that one can often trade off precision for timeliness. It prevents missed deadlines and provides graceful degradation during a transient overload by ensuring that an approximate result of acceptable quality is available whenever the exact result cannot be obtained in time.

The imprecise-computation model used in previous studies [3], [4], [5], [6], [7], [8], [9], [10], [11], [12], [13], [14], [15] assumes that the quality of a task's result depends solely on the time spent by the task to produce the result. Specifically, the input of each task is free of error. If a task terminates prematurely, the result produced by it contains an error that is a nondecreasing function of the processing time of the unexecuted portion. While this model adequately characterizes many real-time applications, there are several others that it does not. Examples include video compression, speech recognition, and radar tracking. In these applications, the quality of a result produced by a task depends also on the quality of the input of the task. When the results produced by some tasks are used as inputs by other tasks, the decision on how much of each task to complete by what time in order for the set of dependent tasks to produce a good overall result should not be made by considering each task independently from the others.

- W. Feng and J.W.-S. Liu are with the Department of Computer Science, University of Illinois at Urbana-Champaign, Urbana, IL 61801.

E-mail:\{feng, janeliu\}@cs.uiuc.edu.

Manuscript received Sept. 9, 1994.

Recommended for acceptance by A.C. Shaw.

For information on obtaining reprints of this article, please send e-mail to: transse@computer.org, and reference IEEECS Log Number $\$ 95622$.
In addition, previous studies on imprecise computation focus on the case where the timing constraints of each task are given. However, the timing constraints that can be derived directly from high-level requirements are typically not that of individual tasks, but rather are timing constraints of sets of tasks. We call such timing constraints endto-end timing constraints. The end-to-end timing constraints over each set of tasks must be met. In contrast, the individual tasks in the set do not have any specific timing constraints, other than those imposed by the end-to-end timing constraints. Thus, we have the freedom to advance and postpone the executions of individual tasks. This freedom gives us an added dimension in the tradeoff between result quality and timing requirements.

In this paper, we extend the imprecise-computation model to account for input error as well as the end-to-end nature of the timing constraints. According to this model, tasks which jointly support a function of the system are dependent. The amount of time required to complete a successor task depends on the quality of the result produced by its predecessor task. Each set of dependent tasks forms a composite task. Composite tasks are independent of each other. The ready time and deadline of each composite task are the end-to-end timing constraints of the component tasks in it. We describe in this paper a two-level approach to scheduling composite tasks. At the high level, the scheduler determines the total amount of time budgeted to each composite task in order for all the composite tasks to meet their deadlines. At the low level, the scheduler distributes the time budgeted for each composite task to its component tasks so as to minimize its output error. The high-level scheduler can use one of several existing algorithms for scheduling independent tasks with precise inputs to determine the time budgets of the composite tasks. We describe here several heuristic algorithms which the low-level scheduler can use to distribute time to component tasks.

The remainder of this paper is organized as follows: Section 2 provides the background information needed for Section 3, which describes the extended imprecise-computation 
model. Section 4 quantifies the effect of input error on the processing time requirements and result quality of dependent component tasks. Section 5 presents a set of heuristic scheduling algorithms which attempt to optimize the overall result quality of each composite task. Section 6 presents the performance of these algorithms. Lastly, Section 7 summarizes our results and presents future work.

\section{BACKGROUND}

Our model is built on the imprecise-computation model used in previous studies [3], [4], [5], [6], [7], [8], [9], [10], [11], [12], [13], [14], [15] which characterizes the workload on a realtime system as a set of preemptable tasks $\mathbf{T}=\left\{T_{1}, T_{2}, \ldots, T_{q}\right\}$. Each task $T_{i}$ is logically decomposed into a mandatory part $M_{i}$ followed by an optional part $O_{i}$ and has the following rational parameters:

- ready time $r_{i}$. The time at which task $T_{i}$ is available for execution,

- deadline $d_{i}$. The time by which task $T_{i}$ must produce a result

- processing time $p_{i}$. The amount of processor time required to execute the task $T_{i}$ to completion,

- mandatory processing time $m_{i}$. The amount of processor time required to execute the mandatory part $M_{i}$ to completion, and

- optional processing time $o_{i}$. The amount of processor time required to execute the optional part $O_{i}$ to completion.

The mandatory part $M_{i}$ must execute to completion in order to produce an acceptable and usable result. The task $T_{i}$ meets its deadline if its mandatory part completes by its deadline. The optional part $O_{i}$ can only execute after the mandatory part $M_{i}$ completes. The optional part may be terminated before it has completed, if necessary, so that the task and other tasks can meet their deadlines.

Our attention is confined to uniprocessor systems. We let $\phi_{i}$ denote the amount of processor time that is assigned to execute the task $T_{i}$, according to a given schedule. A scheduling algorithm works correctly if it never schedules a task before its ready time and if the total time $\phi_{i}$ it assigns to every task $T_{i}$ is no less than the mandatory processing time $m_{i}$. We let $\sigma_{i}=\phi_{i}-m_{i}$ be the amount of time remaining, after the mandatory part $M_{i}$ completes, for the execution of the optional part $O_{i}$. $\sigma_{i}$ may be less than $o_{i}$. Hereafter, by a scheduling algorithm, we mean a correct algorithm, and by a schedule, we mean a valid schedule in which $m_{i} \leq \phi_{i} \leq p_{i}$. We call a schedule in which every task $T_{i}$ is assigned $m_{i}$ or more units of time before its deadline a feasible schedule.

When the assigned processor time $\phi_{i}$ equals $p_{i}$, or equivalently $\sigma_{i}$ equals $o_{i}$, the task $T_{i}$ is said to be precisely scheduled. Otherwise if $\phi_{i}<p_{i}$, the last portion of $T_{i}$ with processing time $p_{i}-\phi_{i}$ is discarded. In a valid schedule, $p_{i}-\phi_{i}$, which is equal to $o_{i}-\sigma_{i}$, is always equal to or less than $o_{i}$. We call $p_{i}-\phi_{i}$ the amount of discarded work and

$$
F_{i}=\frac{o_{i}-\sigma_{i}}{o_{i}}=\frac{p_{i}-\phi_{i}}{o_{i}} \quad \text { for } m_{i} \leq \phi_{i} \leq p_{i}
$$

the fraction of discarded work. ${ }^{1}$ Since $\phi_{i}$ is never in the range $\left[0, m_{i}\right)$, the value of $F_{i}$ in this range is irrelevant; it is assumed to have the value of one for the sake of convenience.

Most existing algorithms [3], [4], [5], [7], [8], [9], [10], [11], [12], [13], [14], [15] for scheduling tasks with optional parts assume that the tasks are monotone. As a monotone task executes longer, the quality of its results improves. Hence, these algorithms seek schedules in which the fraction of discarded work (or some function of this fraction) of each task is as small as possible. Specific performance metrics commonly used by existing algorithms include weighted average of the fractions of discarded work, sum of the fractions of discarded work, maximum fraction of discarded work, and number of discarded optional tasks.

The high-level scheduler described later in this paper makes use of Algorithm $G$ described in [5]. This optimal offline algorithm finds preemptive schedules of independent tasks in which the maximum fraction of discarded work among all tasks is as small as possible. This algorithm in turn uses the optimal algorithm, developed earlier by Shih et al. [11], for scheduling off-line preemptive tasks with arbitrary ready times and deadlines to minimize the sum of the amounts of discarded work over all tasks. While these algorithms allow tasks to be dependent, the possibility of error propagation across dependent tasks was not considered. Shih and Liu [12] has since modified this algorithm to schedule tasks on-line so that the sum of the amounts of discarded work of all tasks accepted by the system is minimized.

The concept of trading off result quality for timeliness has also been independently studied by the artificial intelligence community. Dean and Boddy [16], [17] proposed the use of anytime algorithms in the framework of timedependent planning and decision-making. The execution of an anytime algorithm may be interrupted at any point to return a result whose quality is solely a function of the processing time of the completed portion. Therefore, a task based on the anytime algorithm is an optional task in the imprecise-computation model: The mandatory processing time of such a task is zero.

Zilberstein [18], [19], [20] extended the work of Dean and Boddy by introducing the concept of conditional performance profiles. Conditional performance profiles represent result quality as a function of input quality as well as the processing time spent to produce the result. The extended imprecise-computation model described in the next section resembles Zilberstein's model in this way.

Musliner [21] introduced the concept of any-dimension algorithms - a general class of iterative algorithms. This concept generalizes the anytime algorithm concept by providing guarantees along dimensions other than time. Like an anytime algorithm, an any-dimension algorithm is an iterative algorithm with a termination condition which halts the iteration when some threshold along that dimension is reached. This aspect is not considered here.

Dey, Kurose, and Towsley [22] introduce the notion of reward, which is analogous to error in the imprecisecomputation model or quality in anytime algorithms. They

1. In previous studies on imprecise computation, $E_{i}$ was called the error in the result of task $T_{i}$ when the quality of the result was assumed to be a linear function of the amount of discarded work. 
schedule tasks so that tasks receive increasing reward with increasing service (IRIS). However, their model ignores the dependency of result quality on input quality.

\section{EXTENSIONS TO THE IMPRECISE-COMPUTATION MODEL}

We extend here the imprecise-computation model to characterize applications where inputs provided to tasks may be imprecise. In the extended imprecise-computation model, a workload consists of a set of independent, preemptable composite tasks $\mathbf{T}=\left\{T^{1}, T^{2}, \ldots, T^{q}\right\}$. In particular, the input of each composite task is independent of the output produced by other composite tasks. Each composite task $T^{j}$ consists of $n_{j}$ component tasks $T_{1}^{j}, T_{2}^{j}, \ldots, T_{n_{j}}^{j}$. The parameters $p^{j}, m^{j}$, and $o^{j}$ are the processing time, mandatory processing time, and optional processing time of the composite task $T^{j}$, respectively. They are equal to the sums of the corresponding parameters of the component tasks in $T^{j}$; that is, $p^{j}=\sum_{i=1}^{n_{j}} p_{i}^{j}, m^{j}=\sum_{i=1}^{n_{j}} m_{i}^{j}$, and $o^{j}=\sum_{i=1}^{n_{j}} o_{i}^{j}$, where $p_{i}^{j}, m_{i}^{j}$, and $o_{i}^{j}$ are the processing time, mandatory processing time, and optional processing time of component task $T_{i}^{j}$.

We focus our attention on the type of composite tasks whose component tasks have linear precedence-constraint graphs. The (end-to-end) ready time $r^{j}$ of the composite task $T^{j}$ is the ready time of its first component task $T_{i}^{j} . T_{1}^{j}$ can begin execution at $r^{j}$. $T_{i}^{j}$ can begin execution only after $T_{i-1}^{j}$ completes for $i=2,3, \ldots, n_{j} . T_{i}^{j}$ is the (immediate) successor of $T_{i-1}^{j}$, and the output of $T_{i-1}^{j}$ is the input of $T_{i}^{j}$. The output of the last component task $T_{n_{j}}^{j}$ is the output of the composite task. The (end-to-end) deadline $d^{j}$ of $T^{j}$ is the deadline its last component task $T_{n_{j}}^{j}$. The ready times and deadlines of the intermediate component tasks $T_{2}^{j}, T_{3}^{j}, \ldots, T_{n_{j-1}}^{j}$ are not specified. To capture the end-to-end nature of the timing constraints, we assign the ready times and deadlines of the component tasks to be the same as that of the composite task. This gives us the maximum flexibility in scheduling the component tasks.

Most of this paper deals with the scheduling of component tasks within a given composite task. When there is no ambiguity, we simplify our notation by dropping the superscript $j$ from the component tasks $T_{2}^{j}, T_{3}^{j}, \ldots, T_{n}^{j}$ of the composite task $T^{j}$ and simply call them tasks $T_{1}, T_{2}, \ldots T_{n}$. We assume that all of the component tasks are monotone.

When a component task $T_{i-1}$ is terminated before it is completed, its output contains an error $E_{i-1}$, which is the input error $e_{i}$ of its successor task $T_{i}$. One effect of the input error $e_{i}$ is that the mandatory part $M_{i}$ of $T_{i}$ may be extended in the sense that it now takes an additional $H_{i}\left(e_{i}\right)$ units of processor time beyond $m_{i}$ for $T_{i}$ to produce an acceptable result. We call the $H_{i}\left(e_{i}\right)$ additional time units of the mandatory part the mandatory extension of $T_{i}$, and the concate- nation of the mandatory part $M_{i}$ and the mandatory extension the extended mandatory part $M_{i}^{\prime}$. The amount of processor time needed to execute $M_{i}^{\prime}$ to completion is given by $m_{i}^{\prime}=m_{i}+H_{i}\left(e_{i}\right)$. Throughout this paper, we assume that the mandatory extension $H_{i}\left(e_{i}\right)$ is a monotone nondecreasing function of the input error $e_{i}$ and $H_{i}(0)=0$. Since every component task is monotone, the input error $e_{i}$ of the task $T_{i}$ is a monotone nondecreasing function of the fraction of discarded work $F_{i-1}$ of the predecessor task $T_{i-1}$. Consequently, the mandatory extension of $T_{i}$ is a monotone nondecreasing function of $F_{i-1}$. With a slight abuse of notation, we denote this function by $H_{i}\left(F_{i-1}\right)$, for $i=2,3, \ldots, n$. The mandatory extension of $T_{i}$ is zero if its predecessor task $T_{i-1}$ is assigned $p_{i}$ units of time, i.e., $F_{i-1}=0$, and $H_{i}(0)=0$ for all $i$. We assume that the input error $e_{1}$ to the first component task in every composite task is zero, and hence $H_{1}\left(e_{1}\right)=0$.

The effect on the processing time of the mandatory part can be illustrated by Newton's root-finding method. Suppose that the processing time of the mandatory part is the time it takes to find a root within $10 \%$ of the actual root, and the processing time of the optional part is the time it takes to refine the result of the mandatory part to be within $0.1 \%$ of the actual root. Obviously, if the input to Newton's method is poor (i.e., the predecessor component task provides an input value which is far from the actual root), the mandatory part must execute longer in order to meet the $10 \%$ threshold. This effect is accounted for by the mandatory extension. The optional part stays the same.

Similar examples of extending the mandatory part occur in image and video processing as well. Suppose that the processing time of the mandatory part is the time it takes to display (on a client workstation) a video frame of acceptable quality, and the processing time of the optional part is the time it takes to further enhance the visual quality of the video frame. If during the transmission of a frame to the client, some packets are lost or corrupted; the client must do some image enhancement, and the mandatory part of displaying the video frame is extended correspondingly in order to bring the quality of the video frame up to acceptable standards.

Another possible effect of input error on a task $T_{i}$ is that the processing time of the optional part $O_{i}$ is lengthened. Based on the same argument above, the processing time of the optional part $O_{i}$ of $T_{i}$ is extended by $K_{i}\left(F_{i-1}\right)$ time units to offset the effects of the input error, where $K_{i}(x)$ is a monotone nondecreasing function of $x$ and $K_{i}(0)=0$. The $K_{i}\left(F_{i-1}\right)$ additional time units of the optional part make up the optional extension, and the juxtaposition of the optional part $O_{i}$ and the optional extension is the extended optional part $O_{i}^{\prime}$. The amount of processor time needed to execute $O_{i}^{\prime}$ to completion is given by $o_{i}^{\prime}=o_{i}+K_{i}\left(F_{i-1}\right)$ for $i=2,3, \ldots, n$, and $o_{i}^{\prime}=o_{1}$ because $K_{1}(0)=0$.

Radar tracking is an example of an application where extending the optional part is necessary to compensate for input error. The mandatory part consists of processing the returned radar signal and creating track records by a signal processor. Each track record indicates position and direction of movement of a possible target. The optional part tries to associate these targets with established tracks by a data processor. If the returned radar signal is weak and 
noisy, the amount of time required to process the signal remains more or less the same (i.e., the processing time of the mandatory part stays the same). However, there may be more false returns-records associated with nonexistent targets. As a result, the data processor must spend additional processing time in associating tracks.

In general, both the mandatory part $M_{i}$ and the optional part $O_{i}$ may be extended when a fraction $F_{i-1}$ of the optional part $O_{i-1}$ is discarded and hence the input error $e_{i}$ is nonzero. Specifically, the mandatory part $M_{i}$ is extended by $H_{i}\left(F_{i-1}\right)$ and the optional part $O_{i}$ by $K_{i}\left(F_{i-1}\right)$. An infinite value of $H_{i}\left(F_{i-1}\right)$ means that the input error $e_{i}$ of $T_{i}$ has a fatal effect on $T_{i}: T_{i}$ can never produce an acceptable result no matter how long it executes. Similarly, if $K_{i}\left(F_{i-1}\right)$ is infinite while $H_{i}\left(F_{i-1}\right)$ is finite, the effect of input error can never be erased by executing the optional part $O_{i}^{\prime}$ longer and longer, although the result produced by $T_{i}$ is acceptable. Hereafter, we will refer to $H_{i}\left(F_{i-1}\right)$ and $K_{i}\left(F_{i-1}\right)$ as the mandatory and optional extension functions, respectively.

\section{EFFECTS OF INPUT ERROR}

Again, we consider here composite tasks whose precedence-constraint graphs are linear. For such a composite task, its output error is equal to the output error of the last component task, which, in turn, depends on the output errors of its predecessor component tasks.

Let $\Phi^{j}$ denote the total amount of processor time assigned to a composite task $T^{j}$ by the high-level scheduler. The heuristic algorithms for distributing the total time $\Phi^{j}$ to the component tasks attempt to keep the fraction of discarded work $F_{n}$ of the last component task, and hence the output error $E_{n}$ of this component task and the composite task, as small as possible.

The algorithms presented in subsequent sections are near optimal when the mandatory extension and optional extension of every component task $T_{i}$ are linear functions of the fraction of discarded work $F_{i-1}$ of its immediate predecessor $T_{i-1}$. In particular, they assume that the extension functions of $T_{i}$ are

$$
\begin{array}{ll}
H_{i}\left(F_{i-1}\right)=h_{i} F_{i-1} & \text { for } i=2,3, \ldots, n \\
K_{i}\left(F_{i-1}\right)=k_{i} F_{i-1} & \text { for } i=2,3, \ldots, n
\end{array}
$$

where $h_{i}$ is the mandatory error-scaling factor and $k_{i}$ is the optional error-scaling factor of $T_{i}$. However, the assumption on the linearity of $H_{i}\left(F_{i-1}\right)$ and $K_{i}\left(F_{i-1}\right)$ is not valid in general. We will return in Section 5 to discuss how to approximate arbitrary functions $H_{i}\left(F_{i-1}\right)$ and $K_{i}\left(F_{i-1}\right)$ by linear functions.

Throughout this section, we assume that the mandatory and optional extensions of component tasks $T_{2}, T_{3}, \ldots, T_{n}$ are given by (1) and (2)). The mandatory and optional extensions of the first component task $T_{1}$ of a composite task are zero since the input of a composite task is assumed to be error-free. We first derive the expression for the fraction of discarded work of the last component task, as a function of the amounts of time assigned to the earlier component tasks for the special case where the optional error-scaling factors $k_{i}$ are zero; that is, $k_{i}=0$ for all $i=1,2, \ldots, n_{j}$. We then consider the special case when the mandatory error- scaling factors $h_{i}$ are zero. The expressions for the fraction of discarded work of $T_{i}$ in terms of that of its predecessors will provide clues as to how to distribute the amount of time $\Phi^{j}$ that is assigned to the composite task $T^{j}$ to its component tasks when the mandatory and optional extensions of all component tasks are given by (1) and (2)).

When the mandatory error-scaling factor $h_{i}$ is nonzero and the optional error-scaling factor $k_{i}$ is zero, the amount of time $\phi_{i}$ assigned to each component task $T_{i}$ must be in the range $\left[m_{i}+h_{i} F_{i-1}, m_{i}+h_{i} F_{i-1}+o_{i}\right]$ so that its extendedmandatory part $M_{i}^{\prime}$ can execute to completion. With $\phi_{i}$ constrained to this range, the fraction of discarded work of $T_{i}$ is a linearly decreasing function of $\phi_{i}$, as illustrated by the dotted line in Fig. 1 and stated by the following lemma.

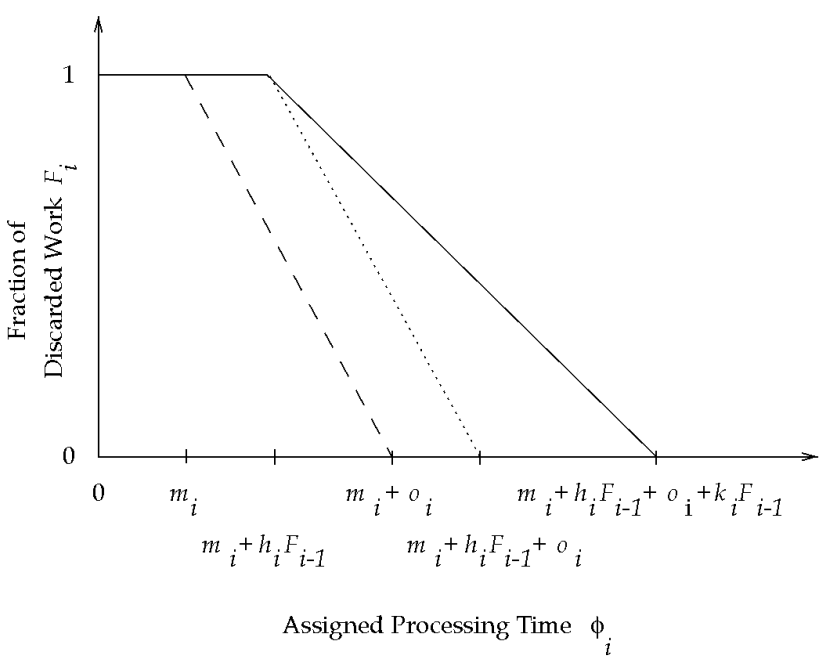

Fig.1. An extended imprecise-computation model.

LEMMA 4.1. The fraction of discarded work $F_{i}$ of a component task $T_{i}$ whose optional error-scaling factor $k_{i}$ is zero is given by

$$
F_{i}=1-\frac{1}{o_{i}}\left(\phi_{i}-m_{i}-h_{i} F_{i-1}\right)
$$

where $m_{i}+h_{i} F_{i-1} \leq \phi_{i} \leq m_{i}+h_{i} F_{i-1}+o_{i}$, when it is assigned $\phi_{i}$ units of time and the fraction of discarded work of its predecessor is $F_{i-1}$.

PROOF. The lemma follows directly from the property of similar triangles.

$$
\frac{F_{i}-0}{\phi_{i}-\left(m_{i}+h_{i} F_{i-1}+o_{i}\right)}=\frac{1-0}{m_{i}+h_{i} F_{i-1}-\left(m_{i}+h_{i} F_{i-1}+o_{i}\right)}
$$

As an example, if $\phi_{i}=m_{i}+h_{i} F_{i-1}$, then there is only enough time to execute the extended-mandatory part $M_{i}^{\prime}$, and the optional part $O_{i}$ is left unexecuted. As a result, $F_{i}=$ 1 . In general, the fraction of discarded work $F_{n}$ of a composite task consisting of $n$ component tasks is given by the following theorem.

THEOREM 4. For a composite task with $n$ component tasks whose optional error-scaling factors are zero, the fraction of discarded work $F_{n}$ of the last component task $T_{n}$ is given by

$$
F_{n}=C_{n}-a_{1} \phi_{1}-a_{2} \phi_{2}-\cdots-a_{n} \phi_{n}
$$

where $\phi_{i}$ is the amount of time assigned to the component task $T_{i}$ for $i=1,2, \ldots, n, C_{n}$ is the constant 


$$
\begin{aligned}
C_{n} & =1+a_{1}\left(m_{1}\right)+a_{2}\left(m_{2}+h_{2}\right)+\cdots+a_{n}\left(m_{n}+h_{n}\right) \\
& =1+\frac{m_{n}}{o_{n}}+\frac{h_{n}}{o_{n}} C_{n-1}
\end{aligned}
$$

and the coefficients $a_{i}$ are given by

$$
\begin{aligned}
a_{1}= & \frac{h_{2} h_{3} \cdots h_{n}}{o_{1} O_{2} \cdots o_{n}}=\frac{h_{2}}{o_{1}} a_{2} \\
a_{2}= & \frac{h_{3} h_{4} \cdots h_{n}}{o_{2} O_{3} \cdots o_{n}}=\frac{h_{3}}{o_{2}} a_{3} \\
& \vdots \\
a_{n-1}= & \frac{h_{n}}{o_{n-1} o_{n}}=\frac{h_{n}}{o_{n-1}} a_{n} \\
a_{n}= & \frac{1}{o_{n}}
\end{aligned}
$$

ProOF. By Lemma 4.1, for a composite task consisting of one component task, its fraction of discarded work is given by

$$
\begin{aligned}
F_{1} & =1-\frac{1}{o_{1}}\left(\phi_{1}-m_{1}\right) \\
& =C_{1}-\frac{1}{o_{1}} \phi_{1}=C_{1}-a_{1} \phi_{1}
\end{aligned}
$$

For a composite task consisting of a chain of two component tasks, the fraction of discarded work $F_{2}$ of $T_{2}$ is equal to

$$
\begin{aligned}
F_{2} & =1-\frac{1}{o_{2}}\left(\phi_{2}-m_{2}-h_{2} F_{1}\right) \\
& =1-\frac{\phi_{2}}{o_{2}}+\frac{m_{2}}{o_{2}}+\frac{h_{2}}{o_{2}}\left(C_{1}-\frac{1}{o_{1}} \phi_{1}\right) \\
& =C_{2}-\frac{h_{2}}{o_{1} o_{2}} \phi_{1}-\frac{1}{o_{2}} \phi_{2}=C_{2}-a_{1} \phi_{1}-a_{1} \phi_{1}-a_{2} \phi_{2}
\end{aligned}
$$

Suppose that the amount of discarded work $F_{n-1}$ for a composite task consisting of a chain of $n-1$ component tasks is

$$
\begin{aligned}
F_{n-1}= & C_{n-1}-\frac{h_{2} h_{3} \cdots h_{n-1}}{o_{1} o_{2} \cdots o_{n-1}} \Phi_{1}-\frac{H_{3} H_{4} \cdots H_{N-1}}{o_{2} o_{3} \cdots o_{n-1}} \Phi_{2}-\cdots \\
& -\frac{1}{o_{n-1}} \Phi_{N-1} \\
= & C_{n-1}-a_{1} \phi_{1}-a_{2} \phi_{2}-\cdots-a_{n-1} \phi_{n-1}
\end{aligned}
$$

Then, the amount of discarded work $F_{n}$ for a composite task with $n$ component tasks is

$$
\begin{aligned}
F_{n}= & 1-\frac{1}{o_{n}}\left(\phi_{n}-m_{n}-h_{n} F_{n-1}\right) \\
= & 1-\frac{\phi_{n}}{o_{n}}+\frac{m_{n}}{o_{n}}+\frac{h_{n}}{o_{n}} \\
& \left(C_{n-1}-\frac{h_{2} h_{3} \cdots h_{n-1}}{o_{1} o_{2} \cdots o_{n-1}} \phi_{1}-\frac{h_{3} h_{4} \cdots h_{n-1}}{o_{2} o_{3} \cdots o_{n-1}} \phi_{2}-\cdots-\frac{1}{o_{n-1}} \phi_{n-1}\right) \\
= & C_{n}-\frac{h_{2} h_{3} \cdots h_{n}}{o_{1} o_{2} \cdots o_{n}} \phi_{1}-\frac{h_{3} h_{4} \cdots h_{n}}{o_{2} O_{3} \cdots o_{n}} \phi_{2}-\cdots-\frac{1}{o_{n}} \phi_{n} \\
= & C_{n}-a_{1} \phi_{1}-a_{2} \phi_{2}-\cdots-a_{n} \phi_{n}
\end{aligned}
$$

Similarly, when the mandatory error-scaling factor $h_{i}$ of a component task $T_{i}$ is zero but the optional error-scaling factor $k_{i}$ is nonzero, the value for $\phi_{i}$ must be in the range $\left[m_{i}, m_{i}+o_{i}^{\prime}\right]$, where $o_{i}^{\prime}=o_{i}+k_{i} F_{i-1}$ is the processing time of the extended-optional part.

LEMMA 4.2. The amount of discarded work $F_{i}$ for a component task $T_{i}$ whose mandatory part has an error-scaling factor $h_{i}$ of zero is given by

$$
F_{i}=1-\frac{\phi_{i}-m_{i}}{o_{i}+k_{i} F_{i-1}}=1-\frac{\sigma_{i}}{o_{i}+k_{i} F_{i-1}}
$$

where $m_{i} \leq \phi_{i} \leq m_{i}+o_{i}+k_{i} F_{i-1}$, when it is assigned $\phi_{i}$ units of time and the fraction of discarded work of its predecessor is $F_{i-1}$.

PROOF. Using Fig. 1, the lemma follows directly from the property of similar triangles.

$$
\frac{F_{i}-0}{\phi_{i}-\left(m_{i}+o_{i}+k_{i} F_{i-1}\right.}=\frac{1-0}{m_{i}-\left(m_{i}+o_{i}+k_{i} F_{i-1}\right)}
$$

THEOREM 4.2. For a composite task with two or more component tasks whose mandatory error-scaling factors $h_{i}$ are zero, the fraction of discarded work $F_{i}$ of component task $T_{i}$ is given by

$$
F_{i}=1-\frac{\sigma_{i}\left(o_{i-1}+k_{i-1} F_{i-2}\right)}{\left(o_{i}+k_{i}\right)\left(o_{i-1}+k_{i-1} F_{i-2}\right)-k_{i} \sigma_{i-1}}
$$

where $\sigma_{i-1}$ and $\sigma_{i}$ are the amounts of time assigned to the optional parts of $T_{i-1}$ and $T_{i}$, respectively, and $F_{i-2}$ is the fraction of discarded work of $T_{i-2}$.

PROOF. From Lemma 4.2,

$$
F_{i}=1-\frac{\sigma_{i}}{o_{i}+k_{i} F_{i-1}}=1-\frac{\sigma_{i}}{o_{i}+k_{i}\left[1-\sigma_{i-1} /\left(o_{i-1}+k_{i} F_{i-2}\right)\right]}
$$

\section{Heuristic Scheduling Algorithms}

We now describe a high-level algorithm for scheduling composite tasks and determining the total amount of time assigned to each task. We then focus on low-level algorithms for distributing the time assigned to each composite task among its component tasks. These algorithms make use of the expressions derived in the previous section to decide how the total amount of time assigned to each composite task should be distributed among its component tasks so as to minimize the output error $E_{n}$ for the composite task. Because the low-level algorithms compute the amounts of time given to the component tasks based on their error-scaling factors, we also present a method for extracting the error-scaling factors.

\subsection{High-Level Scheduling of Composite Tasks}

Since the composite tasks are independent, the quality of the result produced by a composite task is independent of the amounts of time assigned to other composite tasks. Therefore, the problem of scheduling a set of preemptable composite tasks reduces to the problem of scheduling a set of independent preemptable tasks.

Fig. 2 gives a pseudocode description of an algorithm, called S-COMPOSITE, for scheduling composite tasks. (In 
this figure, we use $h^{j}$ to denote the sum $\sum_{i=1}^{n_{j}} h_{i}^{j}$; it is the maximum extended-mandatory processing time.) This algorithm makes use of a modified version of the earliestdeadline-first algorithm (M-EDF), described in [11], and Algorithm G, described in [15]. The M-EDF algorithm treats every composite task as if it were entirely optional and schedules the composite tasks on an earliest-deadline-first basis. It never schedules any composite task after its deadline. In other words, every composite task is terminated at its deadline if it is not completed. It has been shown in [11] that the M-EDF algorithm minimizes the total processing time of the discarded portions of all tasks. Algorithm G distributes the total available processor time as evenly as possible among the tasks. Specifically, for a given set of ready times and deadlines, this algorithm makes the fraction of discarded work of the composite task (i.e., the ratio of the processing time of the unexecuted portion of each composite task to the total optional processing time of all the component tasks in it) as equal as possible to that of the other composite tasks.

Input:

(a) The ready time $r^{j}$ and deadline $d^{j}$ of every composite task $T^{j}$ in $\mathbf{T}$

(b) The total processing time $p^{j}=m^{j}+o^{j}$ and the maximum extended-mandatory processing time $m^{\prime j}=m^{j}+h^{j}$.

(c) The parameters $m_{i}^{j}, o_{i}^{i}, h_{i}^{j}$, and $k_{i}^{j}$ of every component task in every composite task in $T=\left\{T^{1}, T^{2}, \ldots, T^{n}\right\}$

Output:

A feasible schedule of $\mathbf{T}$ or a feasible schedule of a subset of $T$ and the list of composite tasks that cannot be feasibly scheduled.

1. (a) For each composite task $T^{j}, j=1,2, \ldots, q$, set the processing time $P^{j}$ of $T^{j}$ to $p^{j}$.

(b) Use the M-EDF algorithm to find a schedule of $\left\{T^{1}, T^{2}, \ldots, T^{q}\right\}$.

If the resultant schedule is a precise schedule then The output error of every composite task is 0 . Done.

2. (a) For $j=1,2, \ldots, q$, if $P^{j}>m^{\prime \prime}$ Then set $P^{j}=m^{\prime j}$.

(b) Use the M-EDF algorithm to find a schedule of $\left\{T^{1}, T^{2}, \ldots, T^{q}\right\}$.

If the resultant schedule is a precise schedule then Set $\Phi^{j}=P^{j}$ for $j=1,2, \ldots, q$. Go to Step 4 .

3. Use Algorithm G to schedule $\mathbf{T}$.

$\Phi^{j}=$ the total amount of time assigned to $T^{j}$ according the resultant schedule for each composite task in $\mathbf{T}$.

4. (a) For every $j$, use one of the distribution algorithms to distribute the time $\Phi^{j}$ assigned to $T^{j}$ among its component tasks.

(b) Report all infeasible composite tasks and the processing time distribution of all feasible composite tasks.

Fig. 2. Algorithm S-COMPOSITE.

Algorithm S-COMPOSITE has four steps. In Step 1, it tries to schedule every composite task $T^{j}$ precisely. If this step succeeds in finding a precise schedule, the output errors of all the composite tasks are zero, and each component task $T_{i}^{j}$ is assigned $m_{i}^{j}+o_{i}^{j}$ units of time. If Step 1 fails to find a precise schedule of $\mathbf{T}$, no precise schedule of $\mathbf{T}$ exists [11]. Step 2 then tries to precisely schedule every composite task whose optional processing time $o^{j}$ is small compared to its maximum extended-mandatory processing time. It does so in Step 2(a) by reducing the amounts of time assigned to tasks which have relatively large optional processing times. If, in Step 2(b), the M-EDF algorithm finds a precise schedule with this reduction, the composite tasks with small optional processing times are scheduled precisely and have zero output errors. Each composite task whose optional processing times $o^{j}$ is larger than its maximum extended-mandatory processing time $m^{\prime j}$ is assigned a sufficient amount of time to ensure the feasible distribution of its time among its component tasks.

On the other hand, if Step 2(b) fails, we do not know whether we can feasibly schedule $\mathbf{T}$ until we distribute the time $\Phi^{j}$ assigned to each composite task to its component tasks. Step 3 is carried out and finds $\Phi^{j}$ in such a way that the processing times of the unexecuted portions of all composite tasks, measured in terms of fractions of their processing times, are as equal as possible under the constraints imposed by the ready times and deadlines of the composite tasks. Step 4 is then invoked to distribute this time to the component tasks.

For example, suppose that there are two composite tasks with the following parameters:

\begin{tabular}{|c|c|c|c|c|c|}
\hline Task & $m$ & $o$ & $m^{\prime}$ & $r$ & $d$ \\
\hline \hline$T^{1}$ & 15.0 & 14.0 & 26.4 & 0.0 & 28.5 \\
$T^{2}$ & 45.0 & 42.0 & 88.0 & 27.0 & 112.0 \\
\hline
\end{tabular}

Fig. 3 shows the three schedules of the composite tasks produced in Steps 1, 2, and 3 of Algorithm S-COMPOSITE. Because the schedules produced in Step 1 and Step 2 are not precise, Step 3 is carried out, and the fractions of unexecuted optional portions of both tasks are equal to $1 / 14$. The times assigned to these tasks are $\Phi^{1}=28$ and $\Phi^{2}=84$. The processing times of the unexecuted portions of $T^{1}$ and $T^{2}$ are 1 and 3, respectively.

Later, we will expand this example and show that the algorithm used in Step 4 can find a way to distribute the 28 units of time assigned to $T^{1}$ such that the output error is zero and the total time used by its component tasks is less than 28 . The results produced by Step 4 include the amount of unused time for each feasibly scheduled composite task and the additional amount of time required for each infea-

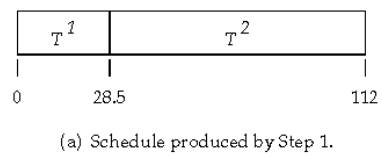

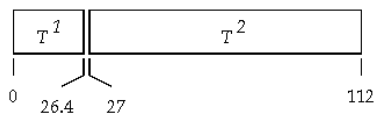

(b) Schedule produced by Step 2.

\begin{tabular}{|c|c|}
\hline$T^{1}$ & $T^{2}$ \\
\hline & \\
\hline & \\
\hline
\end{tabular}

(c) Schedule produced by Step 3 .

Fig. 3. An example to illustrate Algorithm S-COMPOSITE. 
sibly scheduled composite task. We can improve the chance for Algorithm S-COMPOSITE to find a feasible schedule of $\mathbf{T}$ when it fails by carrying out Step 3 and Step 4 again. In the second iteration, the amount of time used by each composite task that is feasibly scheduled in Step 4 in the first iteration is considered to be mandatory. Similarly, the amount of time required by each composite task that is not feasibly scheduled in Step 4 is used as the mandatory processing time of the task in the second iteration.

The time complexity of Steps 1 and 2 is $O(q \ln q)$. The time complexity of Step 3 is $O\left(q^{3}\right)$. The algorithms for distributing time to the component tasks have time complexity $O(n)$. Hence, the time complexity of Algorithm S-COMPOSITE is $O\left(n q+q^{3}\right)$.

\subsection{Low-Level Scheduling to Distribute Processor Time}

The $\Phi$ units of time assigned to a composite task $T$ must be distributed to its $n$ component tasks in such a way that the following constraints are satisfied.

$$
\begin{gathered}
\Phi=\phi_{1}+\phi_{2}+\cdots+\phi_{n} \\
m_{i}+h_{i} F_{i-1} \leq \phi_{i} \leq m_{i}+h_{i} F_{i-1}+o_{i}+k_{i} F_{i-1}
\end{gathered}
$$

We present here five algorithms for this distribution. (Since it is no longer necessary for us to keep track of different composite tasks, we again drop the superscript to simplify our notation.)

Algorithms DIST-M, DIST-M ${ }^{+}$, and DIST-M ${ }^{+}$-ITERATIVE make decisions on the amount of time given to component tasks based primarily on their mandatory error-scaling factors. They are more suited when the processing times of the mandatory extensions of the component tasks are large compared to the processing times of the optional extensions. In contrast, Algorithms DIST-O and DIST-O ${ }^{+}$take into account the effect of the optional error-scaling factors on the output error of the composite task. They should perform better than DIST-M, DIST-M ${ }^{+}$, and DIST-M ${ }^{+}$-ITERATIVE when the processing times of the optional extensions are large in comparison to the processing times of the mandatory extensions.

\subsubsection{Algorithm DIST-M}

Fig. 4 gives the pseudocode description of Algorithm DIST$\mathrm{M}$, an algorithm which distributes processor time to component tasks based solely on the mandatory error-scaling factor; Theorem 4.1 provides the rationale of this algorithm. According to this theorem, when the optional error-scaling factors of all the component tasks are zero (i.e., $k_{i}=0$ for $i=$ $1,2, \ldots, n)$, the fraction of discarded work $F_{n}$ is a linear function of the times $\phi_{i} \mathrm{~s}$ assigned to its component tasks. Since the constraints (6) and (7) on the $\phi_{i}$ s are also linear, the problem of finding an assignment $\left\{\phi_{i}\right\}$ (i.e., the set of times assigned to component tasks) to minimize $F_{n}$, and hence the output error $E_{n}$ of the composite task, is a linearprogramming problem in this special case. We can use a linear-programming package to find $\left\{\phi_{i}\right\}$ if the scheduling is done off-line. However, when $k_{i} \neq 0$, the assignment $\left\{\phi_{i}\right\}$ thus found is not optimal and, as we will see later, may not even be feasible. Algorithm DIST-M and its variances offer better alternatives with lower scheduling overhead because its time complexity is $O(n)$.
Input:

(a) The parameters $m_{i}, o_{i}, h_{i}$, and $k_{i}$ for all $n$ component tasks

(b) The total time $\mathrm{F}$ assigned to the composite task.

Output:

A feasible assignment $\left\{\phi_{i}\right\}$ of time to the component tasks and the amount of unused time $U$, or a report on the failure to find a feasible schedule and the additional time required.

1. If $\Phi \geq \sum_{i=1}^{n}\left(m_{i}+o_{i}\right)$ then

Schedule component tasks precisely by setting

$\phi_{i}=m_{i}+o_{i}$ for $i=1,2,3, \ldots, n$ and $E_{n}=0$.

Done.

2. Set $\phi_{1}=m_{1}$ and $\phi_{i}=m_{i}+h_{i}$ for $i=2,3, \ldots, n-1$.

If $\Phi-\sum_{i=1}^{n-1} \phi_{i} \geq m_{n}+h_{n}+o_{n}+k_{n}$ then

Set $\phi_{n}=m_{n}+h_{n}+o_{n}+k_{n}$.

Report $U=\Phi-\sum_{i=1}^{n} \phi_{i}$.

Done.

3. Set $F_{0}=0$ and $F_{i-1}=1$ for $i=2,3, \ldots, n$

Compute $a_{i} \mathrm{~s}$ from $h_{i} \mathrm{~s}$ and $o_{i} \mathrm{~s}$ for $i=1,2, \ldots, n$, according to (4).

Sort $a_{i} \mathrm{~s}$ in nonincreasing order and put them in the list $L$.

While $L$ is not empty do

$x=$ index of the largest $a_{i}$ in $L$

If $T_{x+1}$ is marked and $x \neq n$ then

$\phi_{x}=m_{x}+h_{x} F_{x-1}$

Else $\phi_{x}=m_{x}+h_{x} F_{x-1}+o_{x}+k_{x} F_{x-1}$

Mark $T_{x}$ and remove $a_{x}$ from $L$.

4. Compute the amount of unused time $U=\Phi-\sum_{i=1}^{n} \phi_{i}$.

If $U<0$, then set

$$
\begin{aligned}
& \phi_{1}=m_{1}, \phi_{i}=m_{i}+h_{i} \text { for } i=2,3, \ldots, n-1 \\
& \phi_{n}=\Phi-\sum_{i=1}^{n-1} \phi_{i} \\
& \text { If } \phi_{n}<m_{n}+h_{n} \text { then } \\
& \text { Report that the algorithm fails and that the amount of } \\
& \quad \text { additional time required is } \\
& \quad \min \left(m_{n}+h_{n}-\phi_{n}-U\right) .
\end{aligned}
$$

Else report unused amount of time $U$ and $\left\{\phi_{i}\right\}$.

Fig. 4. Algorithm DIST-M.

More specifically, Algorithm DIST-M has four steps. The first two steps try to find a feasible assignment in which $T_{n}$ is scheduled to yield a zero output error. If these two steps fail, Step 3 tries to find a good assignment with a small fraction of discarded work $F_{n}$. To see the rationale behind this step, we assume for the moment that $k_{i}=0$ for all $i$. From the expression of $F_{n}$ in Theorem 4.1, we see that this fraction is minimized by choosing the values of $\phi_{i}$ in the following way: The larger the coefficient $a_{i}$ in the sum given by (3), the larger the value of $\phi_{i}$. Unfortunately, because of constraints (6) and (7), this choice of $\phi_{i}$ is not always possible. Because of these constraints and the fact that the $k_{i}$ s are not zero, Step 3, and hence Algorithm DIST-M, is not optimal.

Step 3 tries to find an assignment which yields a small fraction of discarded work $F_{n}$ by making locally optimal or near-optimal decisions as follows. It begins by making the value of $\phi_{x}$, which has the largest coefficient $a_{x}$ among all $a_{i} \mathrm{~s}$ in (3), as large as possible. From (4), we see that $a_{x}>a_{x+1}$ means $h_{x+1}>o_{x}$. In other words, the processing time of the optional part of $T_{x}$ is smaller than the processing time of the maximum mandatory extension of $T_{x+1}$ when the entire op- 
tional part of $T_{x}$ is left unexecuted. Therefore, $\phi_{x}$ is made sufficiently large so that $T_{x}$ can execute to completion. Similarly, because $a_{x}>a_{x-1}$, and hence $h_{x}<o_{x-1}$, less time is required to execute the maximum mandatory extension of $T_{x}$ than the optional portion of $T_{x-1}$. Therefore, $\phi_{x-1}$ is chosen so only the extended-mandatory portion of $T_{x-1}$ completes. This process is repeated until the values of all $\phi_{i}$ s are chosen.

Step 4 checks whether the assignment $\left\{\phi_{i}\right\}$ produced in Step 3 is feasible for the total time $\Phi$ assigned to the composite task. If the total time required by $\left\{\phi_{i}\right\}$ exceeds $\Phi$, then another attempt to find a feasible assignment is made to produce a schedule with the minimum allowable output error. The algorithm fails if this assignment is also infeasible. As a result of Step 4, the amount of time not used by a feasible assignment $\left\{\phi_{i}\right\}$ or the additional amount of time needed for a feasible assignment is reported. The high-level scheduler can make use of this information to reallocate time among composite tasks, e.g., by giving the time not required by one composite task to one(s) that requires more time.

As an illustrative example, we consider the composite task $T^{1}$ in Fig. 3 which consists of a chain of four component tasks: $T_{1}, T_{2}, T_{3}$, and $T_{4}$. (We again suppress the superscript.) The parameters of the component tasks are:

\begin{tabular}{|c|c|c|c|c|}
\hline Task & $m$ & $h$ & $o$ & $k$ \\
\hline \hline$T_{1}$ & 6.4 & 0.4 & 5.0 & 0.0 \\
$T_{2}$ & 4.0 & 4.0 & 2.0 & 0.0 \\
$T_{3}$ & 1.0 & 5.0 & 3.0 & 0.0 \\
$T_{4}$ & 4.0 & 2.0 & 4.0 & 0.0 \\
\hline
\end{tabular}

The total amount of time assigned to $T^{1}$ is $28 . T^{1}$ cannot be precisely scheduled and executed because $\sum_{i=1}^{n}\left(m_{i}+o_{i}\right)=29$. Hence, Step 1 of Algorithm DIST-M fails to find an assignment that yields zero output error. Step 2 assigns $\phi_{1}=6.4$, $\phi_{2}=8.0$, and $\phi_{3}=6.0$ units of time to $T_{1}, T_{2}$, and $T_{3}$, respectively. The remaining time $\Phi-\sum_{i=1}^{3} \phi_{i}=7.6$ is insufficient for $T_{4}$ to execute to completion. Consequently, Step 3 is carried out. Because $a_{4}=\frac{1}{4}, a_{3}=\frac{2}{3} \times a_{4}=\frac{1}{6}, a_{2}=\frac{5}{2} \times a_{3}=\frac{5}{12}$, and $a_{1}=\frac{4}{5} \times a_{2}=\frac{1}{3}$, we have $a_{2}>a_{1}>a_{4}>a_{3}$. In the first iteration, $\phi_{2}=m_{2}+h_{2}+o_{2}=10.0$ is set, and $T_{2}$ is marked. The fraction of discarded work $F_{1}$ of $T_{1}$ is 1 , and the fraction of discarded work $F_{2}$ of $T_{2}$ is 0 . In the second iteration, $a_{1}$ is the largest entry. Because $T_{2}$ is marked and $F_{1}=1, \phi_{1}$ is set to $m_{1}$ $=6.4$. In the third iteration, $a_{4}$ is the largest, and thus $\phi_{4}$ is chosen to be $m_{4}+h_{4}+o_{4}=10$. Because $F_{2}=0$ and $F_{3}=1$ in the fourth iteration, the time required by $T_{3}$ is $m_{3}=1$. Since $\sum_{i=1}^{4} \phi_{i}=6.4+10.0+1.0+10.0=27.4$, the amount of unused time is 0.6 units, and the fraction of discarded work $F_{4}$ of $T_{4}$ is zero. Consequently, the output error is zero.

\subsubsection{Extensions of Algorithm DIST-M}

While Algorithm DIST-M strictly follows Theorem 4.1 when making scheduling decisions, Algorithm DIST- $\mathrm{M}^{+}$only uses it as a guide. The conceptual difference between the two algorithms is in Step 3. Unlike Algorithm DIST-M, Algorithm
DIST-M ${ }^{+}$does not make its scheduling decisions based solely on the $a_{i}$ s. The DIST- $\mathrm{M}^{+}$algorithm goes one step further by checking to see if the total execution time of the pair of tasks $T_{i}$ and $T_{i+1}$ is shortened or lengthened by giving the task $T_{i}$ more processing time. If it is shortened, then task $T_{i}$ is assigned more time; otherwise, it is only allocated enough time to execute the extended-mandatory part. Steps 1, 2, and 4 of this algorithm are the same as the corresponding steps of Algorithm DIST-M; its Step 3 is described in Fig. 5. We note that the purpose of "marking" in this algorithm is different from that in Algorithm DIST-M. Here, a marked task is a task whose fraction of discarded work is zero.

3. Set $F_{0}=0$ and $F_{i-1}=1$ for $i=2,3, \ldots, n$.

Compute $a_{i}$ s from $h_{i}$ s and $o_{i}$ s for $i=1,2, \ldots, n$, according to (4).

Sort $a_{i} \mathrm{~s}$ in nonincreasing order and put them in the list $L$.

While $L$ is not empty do $x=$ index of the largest $a_{i}$ in $L$

If $T_{x+1}$ is marked and $x \neq n$ then

If $o_{x}^{\prime}>h_{x+1} F_{x}+k_{x+1} F_{x}$ then

$\phi_{x}=m_{x}+h_{x} F_{x-1}$

$\phi_{x+1}=m_{x+1}+h_{x+1} F_{x}+o_{x+1}+k_{x+1} F_{x}$

Else

$\phi_{x}=m_{x}+h_{x} F_{x-1}+o_{x}+k_{x} F_{x-1}$

$\phi_{x+1}=m_{x+1}+o_{x+1}$

Mark $T_{x}$

Else $/{ }^{*} T_{x+1}$ is unmarked $* /$

If $o_{x}^{\prime}>h_{x+1} F_{x}$ then

$\phi_{x}=m_{x}+h_{x} F_{x-1}$

$\phi_{x+1}=m_{x+1}+h_{x+1} F_{x}$

Else

$\phi_{x}=m_{x}+h_{x} F_{x-1}+o_{x}+k_{x} F_{x-1}$

$\phi_{x+1}=m_{x+1}$

$\operatorname{Mark} T_{x}$

Remove $a_{x}$ from $L$.

Fig. 5. Step 3 of Algorithm DIST-M+.

The amounts of time required to complete the component tasks may change during the execution of Algorithm DIST-M ${ }^{+}$. If Algorithm DIST-M ${ }^{+}$is applied iteratively, it will produce a schedule as good as or better than a schedule produced by a single pass of Algorithm DIST-M ${ }^{+}$. Therefore, Algorithm DIST-M ${ }^{+}$-ITERATIVE repeatedly applies Algorithm DIST- $\mathrm{M}^{+}$until there are no more changes to the schedule.

\subsubsection{Algorithm DIST-O}

When the processing times of the mandatory extensions are small compared to the processing times of their optional extensions, we expect that DIST-M and its variances are unlikely to perform well. Algorithm DIST-O offers an alternative. The algorithm consists of three steps. Its first two steps are identical to the first two steps of Algorithm DIST-M; its Step 3 is described in Fig. 6. 
Step 3 is carried out when Step 2 fails. In other words, after $m_{i}+h_{i}$ units of time have been assigned to each of the earlier component tasks $T_{i}$, the remaining time $\Phi-$ $\sum_{i-1}^{n-1}\left(m_{i}+h_{i}\right)$ is insufficient for the last component task $T_{n}$ to complete. The decision that must be made is whether to assign this time to $T_{n}$ or an earlier component task or to divide the time between the component tasks in some manner.

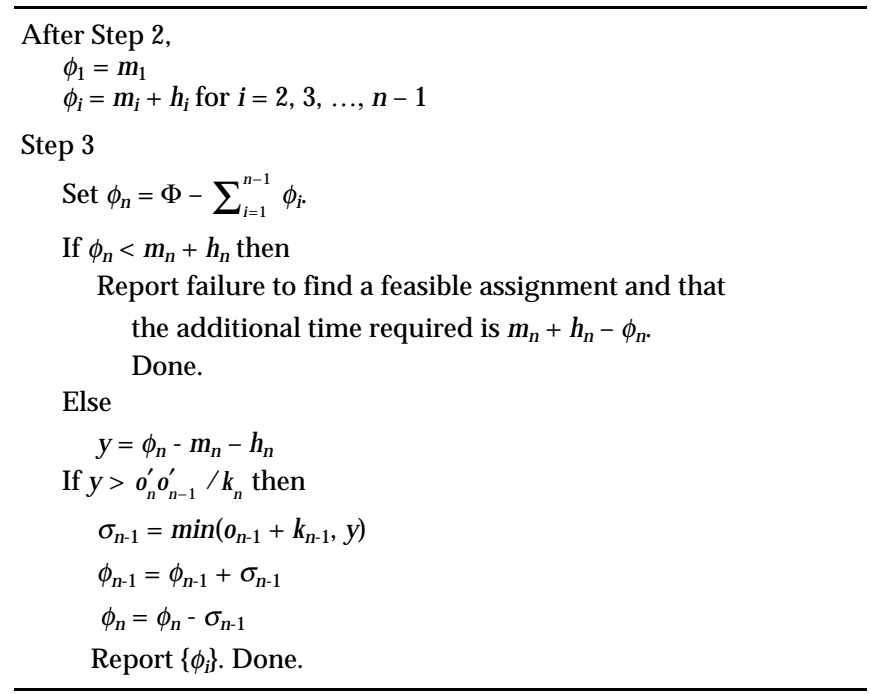

Fig. 6. Step 3 of Algorithm DIST-O.

Algorithm DIST-O is based on the expression of output error given by (5). Like Algorithm DIST-M, the decision made by Algorithm DIST-O is only locally optimal. To understand the reasoning behind the choices made by Step 3, let $y=\Phi-\sum_{i=1}^{n-1}\left(m_{i}+h_{i}\right)$ and $y>0$. We suppose that the $y$ units of time is divided evenly between $T_{n}$ and $T_{n-1}$. According to (5), the fraction of discarded work $F_{n}$ is given by

$$
F_{n}=1-y /\left(2\left[o_{n}^{\prime}-\frac{k_{n} y}{2 o_{n-1}^{\prime}}\right]\right)
$$

where again $o_{n}^{\prime}=o_{n}+k_{n}$ and $o_{n-1}^{\prime}=o_{n-1}+k_{n-1}$. We now ask whether $F_{n}$ can be reduced by assigning $y+\delta$ units of time to $T_{n}$ for $\delta>0$ at the expense of $T_{n-1}$. This question is equivalent to whether the inequality

$$
\left(\frac{y}{2}+\delta\right) /\left(o_{n}^{\prime}-\frac{k_{n}(y / 2-\delta)}{o_{n-1}^{\prime}}\right)>\frac{y}{2} /\left(o_{n-1}^{\prime}-\frac{k_{n} y}{2 o_{n-1}^{\prime}}\right)
$$

is true. A simple algebraic manipulation shows that this inequality holds if

$$
y<\frac{o_{n}^{\prime} o_{n-1}^{\prime}}{k_{n}}
$$

In this case, it is better to give all the remaining time $y$ to $T_{n}$. Otherwise, we give more time to the earlier component task $T_{n-1}$.

\subsubsection{Algorithm DIST-O ${ }^{+}$}

The decision made in Algorithm DIST-O is good only when the processing times of the maximum mandatory extensions of the component tasks are fairly small. In general, we may be able to reduce the output error by distributing the available time to earlier component tasks. Algorithm DIST-O ${ }^{+}$, an enhanced version of Algorithm DIST-O, serves this purpose. This algorithm tries to divide the processing time across all the component tasks; the algorithm is identical to Algorithm DIST-M except that the heuristic guide $a_{i}$ in Step 3 is calculated differently.

The choice of $a_{i}$ is motivated by the expression in (5). According to this equation, when the mandatory error-scaling factors of all the component tasks are zero (i.e., $h_{i}=0$ for $i=$ $1,2, \ldots, n)$, a component task whose optional error-scaling factor and optional processing time are smaller than those of the successor task should be given more time than the successor task when the fraction of discarded work of its predecessor is sufficiently small. Otherwise, the successor task should be given more time. Consequently, we choose $a_{i}$ to be $o_{i+1} k_{i+1} / o_{i} k_{i}$ for $i=1,2, \ldots, n$.

\subsection{Extracting Error-Scaling Factors}

The low-level algorithms for distributing time to component tasks require as input parameters the error-scaling factors of the component tasks. These algorithms cannot be applied directly to component tasks whose extension functions are not in the linear form given by (1) and (2). We now describe how to transform a given set of component tasks with arbitrary mandatory and optional extensions to another set with linear mandatory and optional extensions and thus find the error-scaling factors.

Without loss of generality, suppose that the given mandatory and optional extensions (denoted by $H_{i}^{g}\left(F_{i-1}\right)$ and $K_{i}^{g}\left(F_{i-1}\right)$, respectively) of a component task $T_{i}$ are as shown by the dotted curves in Fig. 7a and 7b, respectively. We approximate each given extension function by two straightline segments; both segments lie entirely above each extension function. These straight-line segments give an upper bound of the values of the extension function at all values of $F_{i-1}$. A valid, feasible schedule based on such straight-line approximations of the extension functions is surely a valid, feasible schedule for the given extension functions.

Specifically, one of the two straight lines is the vertical one at $f_{m}\left(\right.$ or $\left.f_{o}\right)$, where $0<f_{m} \leq 1$ (or $\left.0<f_{o} \leq 1\right)$. We call $f_{i}=\min \left(f_{m}, f_{o}\right)$, the discard threshold of task $T_{i}$. The task $T_{i}$ can never produce a precise result when more than $f_{i}$ fraction of the optional task $O_{i-1}$ of $T_{i-1}$ is left unexecuted. We want to ensure that this condition never occurs for any component task. For this reason, if $f_{i}<1$, we increase the mandatory processing time of $T_{i-1}$ by $\left(1-f_{i}\right) o_{i-1}^{g}$ and decrease the optional processing time of $T_{i-1}$ by $\left(1-f_{i}\right) o_{i-1}^{g}$, where $o_{i-1}^{g}$ denotes the given optional processing time of $T_{i-1}$. In other words, we transform the predecessor task $T_{i-1}$ into one whose mandatory and optional processing times are given by

$$
\begin{aligned}
m_{i-1} & =m_{i-1}^{g}+\left(1-f_{i}\right) o_{i-1}^{g} \\
o_{i-1} & =o_{i-1}^{g}-\left(1-f_{i}\right) o_{i-1}^{g}
\end{aligned}
$$


where $m_{i-1}^{g}$ is the given mandatory processing time of $T_{i-1}$. Our algorithms work with the transformed parameters $m_{i-1}$ and $o_{i-1}$, not the corresponding given parameters.

The allowed range of the fraction of discarded work $F_{i-1}$, based on the computed value $o_{i-1}$ is $[0,1]$. Adopting the straight-line segments with finite slopes in this range, we have the following approximate extension functions:

$$
\begin{aligned}
H_{i}\left(F_{i}\right) & =X F_{i-1} \\
K_{i}\left(F_{i}\right) & =Y F_{i-1}
\end{aligned}
$$

where $X$ and $Y$ are the values defined in Fig. 7. Hence, $h_{i}=$ $X$ and $k_{i}=Y$. Hereafter, we assume that the characteristics of all component tasks are first analyzed, and then the parameters $m_{i}, o_{i}, h_{i}$, and $k_{i}$ of every component task $T_{i}$ in every composite task are derived from the given mandatory and optional processing times and extension functions in the manner described above.

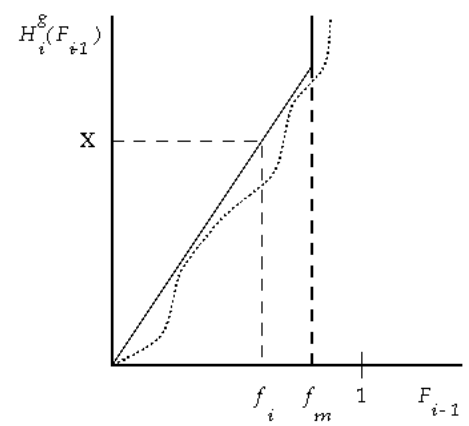

(a)

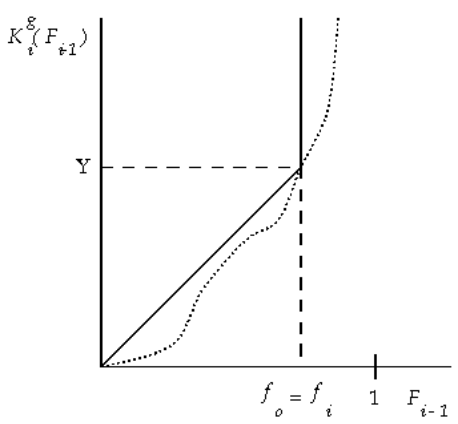

(b)

Fig. 7. Extension functions.

\section{Performance}

We ran a suite of simulation experiments to evaluate the performance of Algorithms DIST-M, DIST-M ${ }^{+}$, DIST-M ${ }^{+}$ITERATIVE, DIST-O, and DIST-O ${ }^{+}$. For convenience, we call the five algorithms collectively as DIST-* algorithms. In each experiment, we randomly generated a composite task and each of its component tasks. For each component task, the mandatory and optional processing times (i.e., $m_{i}$ and $o_{i}$ ) and the mandatory and optional error-scaling factors (i.e., $h_{i}$ and $k_{i}$ ) were randomly chosen from their respective probability distributions; we isolated the effect of these parameters by keeping all other parameters fixed. (For example, the number of component tasks in a composite task was fixed at 8.) For each composite task, the parameters $m_{i}, h_{i}, o_{i}$, and $k_{i}$ of each component task $T_{i}$ were chosen from either uniform distributions or bimodal distributions. We then applied the five algorithms on each composite task and found the fractions of discarded work of the last component task produced by them. For a given algorithm, the lower the fraction of discarded work, the better the performance.

\subsection{Uniform Distribution}

Table 1 shows the performance of the DIST-* algorithms found in four representative experiments where the parameters $m_{i}, h_{i}, o_{i}$, and $k_{i}$ of each $T_{i}$ were all chosen from uniform distributions. Each column corresponds to a composite task whose component-task parameters are selected from either the uniform distribution in the range $[0,10)$ or the uniform distribution in the range $[0,100)$. We call the distribution with the range $[0,10)$ to be the "small" uniform distribution. So, when the parameter $h_{i}$ of all component tasks are chosen from the "small" distribution and $m_{i}, o_{i}$, and $k_{i}$ from the larger distribution, namely the one with range $[0,100)$, we label the appropriate column with "small $h$ " as exemplified by the second column of each experiment in Table 1.

The numerical entries in Table 1 are either in roman or italic font. The first two steps of all five DIST-* algorithms are the same; these steps try to find a feasible scheduling assignment which yields zero output error. When either Step 1 or 2 succeed, the results are listed in roman font. If either of these steps fail, then the succeeding steps distinguish the individual DIST-* algorithms. The results for the composite tasks which benefit from these distinguishing steps are in italics. If none of the steps result in a feasible schedule, then the composite task is said to be unschedulable (uns).

When the expected values of the $h_{i}$ parameters are small, the five algorithms perform almost identically. Small $h_{i}$ parameters imply that compensating for the input error of a component task can be much cheaper timewise than scheduling its predecessor tasks precisely. As a result, the best approach to schedule these types of tasks in general is to schedule only the extended mandatory parts of all but the last component task and then compensate for the accumulated error by giving the last component task as much time as possible. This approach is what Step 2 of all the DIST-* algorithms does, thus explaining the near-identical performance.

The only instance where the algorithms do not perform identically when $h_{i}$ are small is when $o_{i}$ and $k_{i}$ are small as well. In this case, the approach described above takes about as much time as scheduling the component tasks precisely because all the optional parameters are small. In other words, the time given to the last component task to compensate for error in the mandatory parts (i.e., the mandatory extensions) and for the accumulated error of its predecessors is comparable to the amount of time it takes to simply execute all the shorter optional parts entirely to begin with. Because neither effect is pronounced, the heuristics in Steps 3 or 4 of the DIST-* algorithms can come into play and possibly produce better schedules. For instance, for small $h_{i}, o_{i}$, and $k_{i}$ in Experiment 1, DIST-M ${ }^{+}$and DIST-M ${ }^{+}-$ ITERATIVE achieve the fraction of discarded work of 0.211 and perform better than the other algorithms. 
TABLE 1

FRACTION OF DISCARDED WORK—UNIFORMLY DistRIBUTED PARAMETERS

Experiment 1

\begin{tabular}{|c|c|c|c|c|c|c|c|c|c|c|c|c|c|c|c|}
\hline \multirow{2}{*}{$\begin{array}{l}\text { Algorithm } \\
\text { DIST- } \\
\end{array}$} & \multicolumn{15}{|c|}{ small } \\
\hline & $m, h, o, k$ & h & $h, k$ & $h, 0$ & $h, 0, k$ & $k$ & 0 & $o, k$ & $m$ & $m_{1} h$ & $m, h, k$ & $m, h, o$ & $m, k$ & $m, o$ & $m, o, k$ \\
\hline M & .991 & .420 & 489 & .874 & .861 & $.16 \mathrm{t}$ & .000 & .000 & uns & .981 & .980 & .883 & .988 & 000 & . 000 \\
\hline $\mathrm{M}^{+}$ & .850 & .420 & .486 & .874 & .211 & $.09 t$ & .000 & .000 & .969 & $9 \$ 1$ & .980 & .883 & .268 & .000 & .000 \\
\hline $\mathrm{M}^{+}$-ITER & .850 & .420 & .488 & .874 & .211 & $.09 t$ & .000 & .000 & .968 & .881 & .980 & .883 & .268 & .0000 & .000 \\
\hline 0 & .991 & 420 & .489 & 874 & 36.1 & uns & .000 & .000 & uns & .981 & .980 & .880 & Uns & .000 & .000 \\
\hline $\mathrm{o}^{+}$ & 901 & .420 & -489 & .874 & .361 & uns & .000 & .000 & Uns & .981 & .980 & .883 & uns & .000 & .000 \\
\hline
\end{tabular}

Experiment :

\begin{tabular}{|c|c|c|c|c|c|c|c|c|c|c|c|c|c|c|c|}
\hline \multirow{2}{*}{$\begin{array}{l}\text { Algorithen } \\
\text { DIST- }\end{array}$} & \multicolumn{15}{|c|}{ small } \\
\hline & $m, h, 0, h$ & $h$ & $h, k$ & $h, 0$ & $h, 0, k$ & $\bar{k}$ & 0 & $0, k$ & $m$ & $m_{1} h$ & $m, h, h$ & $m, h, 0$ & $\pi \pi, k$ & $\mathbf{m , 0}$ & $m, 0, k$ \\
\hline$M$ & .144 & .898 & .882 & .922 & .894 & .085 & uns & يास & .026 & +873 & .853 & .943 & .080 & นกร & uns \\
\hline$M^{+}$ & .055 & .898 & .882 & .922 & 795 & .085 & uns & uns & .000 & .873 & .853 & .943 & .080 & uns & uns \\
\hline $\mathbf{M}^{+}$ITER & .055 & 898 & .882 & .922 & .795 & .0 .85 & uns & Lns & .000 & .873 & .858 & .943 & .080 & นกs & uns \\
\hline 0 & uns & .898 & .882 & .922 & .924 & uns & uns & uns & uกา & .873 & .853 & .943 & uns & uns & uns \\
\hline $\mathrm{o}^{+}$ & uns & .898 & .882 & .922 & .924 & uns & uns & Luns & a1าs & 873 & 858 & .943 & uns & uns & uns \\
\hline
\end{tabular}

Experiment :

\begin{tabular}{|c|c|c|c|c|c|c|c|c|c|c|c|c|c|c|c|}
\hline \multirow{2}{*}{$\begin{array}{c}\text { Algorithen } \\
\text { DIST: }\end{array}$} & \multicolumn{15}{|c|}{ small } \\
\hline & $m, h, o, k$ & $h$ & $h, h$ & $h, 0$ & $h, o, k$ & $\boldsymbol{k}$ & 0 & $0, k$ & $\mathrm{~m}$ & $m, h$ & $m, h, h$ & $\boldsymbol{m}, \overline{\mathbf{h}}_{\boldsymbol{t}} \mathrm{o}$ & $m, k$ & $\pi n, 0$ & $m, o, k$ \\
\hline$M$ & .572 & .968 & .677 & 121 & .968 & .914 & , 000 & 000 & uns & .905 & .883 & .681 & .878 & .000 & .000 \\
\hline $\mathrm{M}^{+}$ & .994 & 968 & +677 & .121 & .968 & $.9 I_{4}$ & .000 & .000 & .876 & .905 & .883 & .681 & .872 & .000 & .000 \\
\hline$M^{+t}-1 T E R$ & .394 & .988 & .677 & .121 & .968 & .914 & .000 & .000 & .876 & .905 & .883 & .681 & $87 z$ & .000 & .000 \\
\hline 0 & .572 & .968 & .677 & .121 & .968 & tens & .000 & .000 & uns & .905 & .883 & .681 & $87 t$ & .000 & .000 \\
\hline $0^{+}$ & .572 & 968 & .677 & .121 & .968 & nns & .000 & .ón & uns & .905 & .883 & $.68_{1}$ & .870 & .000 & .000 \\
\hline
\end{tabular}

Bapperiment 4

\begin{tabular}{|c|c|c|c|c|c|c|c|c|c|c|c|c|c|c|c|}
\hline \multirow{2}{*}{$\begin{array}{l}\text { Aigorithm } \\
\text { DIST- }\end{array}$} & \multicolumn{15}{|c|}{ small } \\
\hline & $\mathrm{m}, h, 0, k$ & $h$ & $h, k$ & $\hat{h, 0}$ & $h, o, k$ & $\boldsymbol{k}$ & $a$ & $o_{1} k$ & $m$ & $m, h$ & $m, h, h$ & $m, h, 0$ & $m, k$ & $\pi, 0$ & $\boldsymbol{m}, o, k$ \\
\hline $\mathbf{M}$ & .208 & +985 & $\begin{array}{r}139 \\
\end{array}$ & .912 & .065 & .878 & ung & นns & .018 & .886 & .742 & .967 & .908 & uns & Lns \\
\hline $\mathrm{M}+$ & .208 & 985 & .139 & .912 & .170 & .878 & uns & uns & .018 & .886 & .742 & .987 & .902 & uns & uns. \\
\hline $\mathrm{M}^{+}$-ITER & .008 & 985 & .139 & .912 & -170 & .878 & uns & uns & .018 & +886 & .742 & 967 & .902 & uns & uns \\
\hline 0 & .924 & 985 & .139 & .912 & .742 & nus & uns & uns & .734 & 886 & .742 & .987 & uns & uns & uns \\
\hline $\mathrm{O}^{+}$ & .984 & +985 & +139 & .912 & .742 & uns & Uns & uns & .734 & .886 & .742 & .967 & uns & นлs & uns \\
\hline
\end{tabular}

In general, whenever $h_{i}$ are small and $o_{i}$ are large, the better approach is to schedule only the extended mandatory parts and then compensate for the accumulated error by giving the last component task as much time as available. On the other hand, whenever the $o_{i} \mathrm{~s}$ or $k_{i} \mathrm{~s}$ are small, the better approach is to schedule all the component tasks precisely. The performance of the five heuristics seen in all the experiments where the $o_{i} \mathrm{~s}$ or $k_{i} \mathrm{~s}$ are small supports this hypothesis. The heuristic steps in the DIST-* algorithms effectively "reconcile" these opposing approaches and determine how much of each scheduling approach to use in finding an assignment for each set of parameter values.

In summary, Algorithms DIST-M ${ }^{+}$and DIST-M ${ }^{+}$ITERATIVE perform better than the other algorithms in most cases; however in some cases, they do not. For example, in Experiment 4 when $h_{i}, o_{i}$, and $k_{i}$ are small, DIST-M performs better than both DIST-M ${ }^{+}$and DIST-M ${ }^{+}-$ ITERATIVE because the locally optimal decisions made by DIST-M ${ }^{+}$and DIST-M ${ }^{+}$-ITERATIVE were not globally optimal. Finally, the results of this set of experiments do not distinguish the performance of DIST- $\mathrm{M}^{+}$and DIST-M ${ }^{+}-$ ITERATIVE.

\subsection{Bimodal Distribution}

Table 2 shows the results obtained in four representative experiments where the parameters $m_{i}, h_{i}, o_{i}$, and $k_{i}$ were chosen from either uniform or bimodal distributions. Specifically, in each experiment, the parameters of each component task were selected from either a uniform distribution in the range $[0,100)$ or a bimodal distribution in the ranges $[0,10)$ or $[90,100)$. (The bimodal probability density function is equal to $1 / 20$ in the ranges $[0,10)$ and $[90,100)$ and is equal to zero elsewhere.) For example, when the $h_{i}$ parameters are chosen from the bimodal distribution and $m_{i}, k_{i}$, and $o_{i}$ are chosen from the uniform distribution, we label the appropriate column with "bimodal $h$. .

The results in Table 2 show that the different heuristics used in the different DIST-* algorithms lead to a larger difference in performance when all or some of the task parameters are bimodally distributed. The reason is that Steps 1 and 2 do not often succeed for tasks with bimodally distributed parameters. For instance, while column 2 of Table 1 shows that when $m_{i}, o_{i}$, and $k_{i}$ are large and $h_{i}$ is small, all five DIST-* algorithms achieve the same result; the corresponding case of large $m_{i}, o_{i}$, and $k_{i}$ chosen from the same uniform distribution but $h_{i}$ chosen from the bimodal distri- 
TABLE 2

FRACTION OF DISCARDED WORK-BIMODAL AND UNIFORMLY DISTRIBUTED PARAMETERS

Experiment I

\begin{tabular}{|c|c|c|c|c|c|c|c|c|c|c|c|c|c|c|c|}
\hline \multirow{2}{*}{$\begin{array}{c}\text { Algorithm } \\
\text { DIST- }\end{array}$} & \multicolumn{15}{|c|}{ bimodal } \\
\hline & $m, h, o, k$ & $h$ & $h, k$ & $h, \mathrm{o}$ & $h_{1}, 0, k$ & $\bar{k}$ & 0 & $o, k$ & $m$ & $\pi, \bar{h}$ & $\mathrm{~m}, h, k$ & $m, h, a$ & $m, k$ & $m, 0$ & $m_{1}, 0, k$ \\
\hline$M$ & .000 & .920 & .998 & ung & .225 & .948 & uns & $.99 s$ & .834 & 950 & .958 & uns & .668 & uns & 2.961 \\
\hline $\mathrm{HI}^{+}$ & .000 & .894 & .932 & .946 & .844 & .948 & uns & 990 & .834 & .855 & .958 & .569 & .669 & uns & .898 \\
\hline$M^{+}$-ITEEL & .000 & .294 & .992 & .946 & .846 & .948 & uns & .990 & .834 & 865 & .858 & .569 & .669 & uns & .898 \\
\hline$o$ & шп8 & .280 & uns & uns & uns & .948 & uns & uns & .834 & .950 & uns & เาก & .872 & uns & uns \\
\hline $0^{+}$ & uns & .920 & uns & uns & uns & .948 & uns & uns & .834 & .850 & uns & uns & .872 & uns & uns \\
\hline
\end{tabular}

Experiment 2

\begin{tabular}{|c|c|c|c|c|c|c|c|c|c|c|c|c|c|c|c|}
\hline \multirow{2}{*}{$\begin{array}{l}\text { Algorithen } \\
\text { DIST- }\end{array}$} & \multicolumn{15}{|c|}{ binacdel } \\
\hline & $m, h, o, k$ & $h$ & $h_{,}, k$ & $h_{,}, o$ & $h_{1}, 0, k$ & $h$ & 0 & $o, k$ & $m$ & $m, h$ & $m, h, k$ & $m, h, d$ & $\pi, k$ & $m, o$ & $\pi, 0, k$ \\
\hline $\mathrm{M}$ & .080 & uns & .958 & .789 & .816 & .797 & uns & .946 & 990 & urrs & .670 & .717 & .804 & uns & .870 \\
\hline $\mathrm{M}^{+}$ & .058 & .899 & .958 & 899 & $.855_{4}$ & .797 & this & .946 & $99_{1}$ & .955 & .570 & .824 & .804 & ums & .870 \\
\hline$M^{+}$ITER & .020 & 899 & .958 & 789 & .816 & .787 & uns & .946 & $.99 I$ & .955 & .670 & .714 & .804 & uns & 870 \\
\hline 0 & uns & uns & uns & uns & uns & uns & uns & uns & שns & यก8 & uns & uns & uns & uns & uns \\
\hline $\mathrm{o}^{+}$ & uns & uns & uns & uns & นงa & ants & uns & uns & uns & uns & uns & uns & uns & uns & uns: \\
\hline
\end{tabular}

Experinent 9

\begin{tabular}{|c|c|c|c|c|c|c|c|c|c|c|c|c|c|c|c|}
\hline \multirow{2}{*}{$\begin{array}{l}\text { Algorithrn } \\
\text { DIST- }\end{array}$} & \multicolumn{15}{|c|}{ binodal } \\
\hline & $\mathbf{m}, h, o, k$ & $h$ & $h, h$ & $h_{9}, 0$ & $h, o, k$ & $k$ & 0 & $0, k$ & $m$ & $m, h$ & $m, h, h$ & $m, h_{y} o$ & $m_{1} k$ & $m, o$ & $m, 0, k$ \\
\hline $\mathbf{M}$ & .154 & .892 & .154 & .8.99 & .062 & .944 & UDE & .887 & .735 & .787 & .808 & .024 & .932 & uns & .642 \\
\hline $\mathrm{M}^{+}$ & .154 & .858 & .154 & uns & .062 & .944 & .896 & .887 & .735 & .787 &.$A 08$ & .914 & .832 & .791 & .642 \\
\hline $\mathrm{M}^{+}$-ITER & .154 & .892 & .154 & .751 & .062 & .944 & .826 & .887 & .735 & .787 & .808 & .516 & .932 & 791 & .642 \\
\hline 0 & แมธ & .942 & $\operatorname{Lns}$ & une & uns & .944 & Uas & uns & .735 & .897 & uns & uns & .982 & uns & uns \\
\hline$\varphi^{+}$ & uns & .942 & uns & uns & thas & .944 & UTzB & uns. & .735 & .897 & ung & uns & .932 & uns & uns \\
\hline
\end{tabular}

Experiment 4

\begin{tabular}{|c|c|c|c|c|c|c|c|c|c|c|c|c|c|c|c|}
\hline \multirow{2}{*}{$\begin{array}{l}\text { Algorithrn } \\
\text { DIST- }\end{array}$} & \multicolumn{15}{|c|}{ bimodel } \\
\hline & $m, h_{1}, \theta_{1}, k$ & $h$ & $h, h$ & $h_{40}$ & $h, 0, k$ & $k$ & 0 & $0, k$ & $\pi$ & $m, h$ & $m h, h, k$ & $m, h, o$ & $m, k$ & $m, 0$ & $m, o, k$ \\
\hline $\mathbf{M}$ & .592 & .559 & .865 & uns & บกs & .932 & uns & .849 & .859 & .895 & $.68 ?$ & uns & .873 & uns & .879 \\
\hline $\mathbf{m}^{+}$ & .698 & .559 & .885 & uns & .947 & .982 & .814 & .849 & .859 & .896 & .687 & uns & .873 & .790 & $g 7 s$ \\
\hline $\mathrm{M}^{+}$-ITER & .598 & .559 & .865 & .721 & .947 & .982 & .814 & .849 & .859 & .896 & .687 & .687 & .873 & .790 & .879 \\
\hline 0 & ung & .808 & UกE & uns & uns & .937 & uns & uns & .859 & .909 & uns & uns & .873 & uns & uns \\
\hline$o^{+}$ & uns & .808 & UDE, & uns & uns & .932 & บns & uns & .859 & .209 & uns & uns & .873 & uns & uns \\
\hline
\end{tabular}

bution (column 2 of Table 2) shows that the DIST-M $\mathrm{M}^{+}$and DIST-M ${ }^{+}$-ITERATIVE algorithms produce better results than the other algorithms. The bimodal mixture of small and large $h_{i}$ makes the approaches taken by Step 1 and Step 2 less effective, thus allowing the heuristics of the DIST-M ${ }^{+}$ and DIST-M ${ }^{+}$-ITERATIVE algorithms to be applied more frequently and leading to better results. That Steps 1 and 2 fail more frequently for tasks with bimodally distributed parameters is demonstrated by the larger number of italicized entries in Table 2.

As in Table 1 when $o_{i}$ or $k_{i}$ are small, Table 2 shows that when $o_{i}$ or $k_{i}$ are bimodally distributed, the approach of scheduling all the component tasks precisely is still a good one. However, it is no longer as good as in the case when all task parameters are uniformly distributed; this is due mainly to the fact that fewer of the $o_{i}$ and $k_{i}$ parameters are small in the bimodal distribution when compared to the uniform distribution for a given composite task. Because the sample of $o_{i}$ and $k_{i}$ parameters is no longer uniformly distributed, the heuristics potentially have more opportunities to tradeoff the processing times of the mandatory and optional extensions of the component tasks, thus producing better results.
In summary, as in Table 1 , Algorithms DIST- $\mathrm{M}^{+}$and DIST- $\mathrm{M}^{+}$-ITERATIVE generally perform better than the other algorithms when some parameters are bimodally distributed. Furthermore, DIST-M ${ }^{+}$-ITERATIVE always performed as well as or better than DIST-M ${ }^{+}$.

\section{SUMmARY AND FUtURE WORK}

In this paper, we extended the imprecise-computation technique to account for input error and end-to-end timing constraints and developed five heuristic scheduling algorithms-DIST-M, DIST-M ${ }^{+}$, DIST-M ${ }^{+}$-ITERATIVE, DIST-O, and DIST- $\mathrm{O}^{+}$- to minimize the output error of each composite task in a real-time system. The algorithms all have time complexity $O(n)$. Although our initial intuition indicated that the DIST-O and DIST-O ${ }^{+}$algorithms would perform better when the processing times of mandatory extensions were small compared to that of the optional extensions, this turned out to be false. For the suite of simulation experiments that were run to evaluate these algorithms, the DIST-M algorithm and its variants always performed as well as or better than either Algorithm DIST-O or Algorithm DIST-O ${ }^{+}$. This makes sense because the optional ex- 
tension does not have to be executed in any given schedule whereas the mandatory extension must always be executed. When an optional extension is "too long" (e.g., $k_{i}$ and/or $o_{i}$ large), the algorithms choose not to schedule it. Furthermore, when the mandatory extensions are small (i.e., $h_{i}$ and/or $m_{i}$ small), it is easier to compensate for the accumulated error produced by earlier component tasks by scheduling the last component task as much as possible.

As stated in Section 5, we could have used a linearprogramming solver to do time distribution for the special case where the optional error-scaling factors are all zero. However, when the linear-programming solutions are applied to the composite tasks in the experimental suite (all of which have nonzero optional error-scaling factors), the solution provided by the linear-programming solver gives us an infeasible time distribution for every composite task generated in our experiments.

There are many natural extensions of this work including the generation of other precedence constraint graphs and more accurate piecewise linear approximations of the extension functions. For example, we are evaluating the performance of our algorithms when the extension functions of component tasks are concave and convex. Yet another extension would make more of a global decision in assigning time to the component tasks of a composite task. Specifically, when the low-level algorithm is unable to schedule the component tasks of a composite task within its allotted time, the lowlevel algorithm could provide feedback to the high-level algorithm about its additional processing-time needs. If the high-level algorithm finds other composite tasks that do not require the entirety of their assigned processing times, the unschedulable composite task could borrow time from them in order to become schedulable.

\section{ACKNOWLEDGMENTS}

This work was supported in part by the Office of Naval Resarch under Contract No. N00014-92-J-1146 and the Air Force Office of Scientific Research under Contract No. F49620-93-1-0060.

\section{REFERENCES}

[1] A.L. Liestman and R.H. Campbell, "A Fault-Tolerant Scheduling Problem," IEEE Trans. Software Eng., vol. 12, no. 10, pp. 1,0891,095, Oct. 1986.

[2] J. Blazewicz and G. Finke, "Minimizing Mean Weighted Execution Time Loss on Identical and Uniform Processors," Information Processing Letters, vol. 24, no. 3, pp. 259-263, Mar. 1987.

[3] K.-J. Lin and S. Natarajan, "Concord: A System of Imprecise Computations," Proc. Compsac, IEEE , pp. 75-81, Oct. 1987.

[4] K.-J. Lin, S. Natarajan, and J.W.-S. Liu, "Imprecise Results: Utilizing Partial Computations in Real-Time Systems," Proc. Eighth Real-Time Systems Symp., IEEE , Dec. 1987.

[5] J.W.-S. Liu, K.-J. Lin, and C.L. Liu, "A Position Paper for the 1987 IEEE Workshop on Real Time Operating Systems," Proc. Workshop on Real-Time Operating Systems, IEEE, May, 1987.

[6] E.K.P. Chong and W. Zhao, "Performance Evaluation of Scheduling Algorithms for Imprecise Computer Systems," Technical Report S5001, Dept. of Computer Science, Univ. of Adelaid, Sept. 1988.

[7] J.Y.-T. Leung, T.W. Tam, C.S. Wong, and G.H. Young, "Minimizing Mean Flow Time with Error Constraints," Proc. 10th Real-Time Systems Symp., IEEE, pp. 2-11, Dec. 1989.
[8] J.-Y. Chung, J.W.-S.Liu, and K.-J. Lin, "Scheduling Periodic Jobs that Allow Imprecise Results," IEEE Trans. Computers, vol. 19, no. 9, pp. 1,156-1,173, Sept. 1990.

[9] J.Y.-T. Leung and C.S. Wong, "Minimizing the Number of Late Tasks with Error Constraints," Proc. 11th Real-Time Systems Symp., IEEE, pp. 32-40, Dec. 1990.

[10] J.W.-S. Liu, K.-J. Lin, W.-K. Shih, A.C.-S. Yu, J.-Y. Chung, and W. Zhao, "Algorithms for Scheduling Imprecise Computations," Computer, vol. 24, no. 5, pp. 58-68, May 1991.

[11] W.-K. Shih, J.W.-S. Liu, and J.-Y. Chung, "Algorithms for Scheduling Imprecise Computations to Minimize Total Error," SIAM J. Computing, vol. 20, no. 3, July 1991.

[12] W.-K. Shih and J.W.-S. Liu, "On-Line Scheduling of Imprecise Computations to Minimize Error," Proc. 13th Real-Time Systems Symp., IEEE, Dec. 1992.

[13] I.K. Cheong, "Scheduling Imprecise Hard Real-Time Jobs with Cumulative Error, "Technical Report UIUCDCS-R-92-1758, Dept. of Computer Science, Univ. of Illinois at Urbana-Champaign, June 1992.

[14] W. Zhao, J.W.-S. Liu, and S. Verbsky, "Imprecise Scheduling in Multiprocessor Systems, Proc. Fifth IEEE Int'l Conf. Parallel and Distributed Computing Systems, Sept. 1992.

[15] W.-K. Shih and J.W.-S. Liu, "Algorithms for Scheduling Imprecise Computations with Timing Constraints to Minimize Maximum Error," to appear in IEEE Trans. Computers.

[16] T. Dean and M. Boddy, "An Analysis of Time-Dependent Planning, " Proc. AAAI, pp. 49-54, 1988.

[17] M. Boddy and T. Dean, "Deliberation Scheduling for Problem Solving in Time-Constrained Environments," Artificial Intelligence, vol. 67 , pp. $245-285,1994$

[18] S. Zilberstein, "Constructing Utility-Driven Real-Time Systems Using Anytime Algorithms," Proc. First Workshop on Imprecise and Approximate Compuation, IEEE, Dec. 1992.

[19] S. Zilberstein, "Operational Rationality Through Compilation of Anytime Algorithms," PhD thesis, Dept. of Computer Science, Univ. of California at Berkeley, 1993.

[20] S. Zilberstein, "Optimizing Decision Quality with Constract Algorithms, Proc. IJCAI-95, 1995.

[21] D.J. Musliner, "CIRCA: The Cooperative Intelligent Real-Time Control Architecture," PhD thesis, Dept. of Computer Sicence and Engineering, Univ. of Michigan, 1993.

[22] J. Dey, J. Kurose, and D. Towsley, "On-Line Processor Scheduling for a Class of Iris Real-Time Tasks, to appear in IEEE Trans. on Computers.

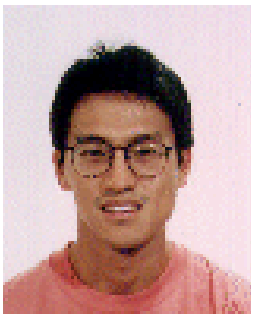

Wu-chun Feng (S'88-M'96) received a BS degree in computer engineering and a BS (Honors) degree in music from The Pennsylvania State University in 1988; an MS degree in computer engineering from The Pennslvania State University in 1990; and a PhD degree in computer science from the University of Illinois at UrbanaChampaign in 1996. He is currently a visiting assistant professor at the University of Illinois at Urbana-Champaign and a researcher for Vosaic Corporation. He is the co-author of Fortan at the Keyboard (Kendall/Hunt Publishing) and has published numerous papers in a wide range of areas that include real-time systems and networks, video/image processing, parallel processing (compilers and applications), and natural-language processing. His current research is focused on the streaming of video and audio over the Internet. Prior to joining the University of Illinois in 1996, he was a teaching fellow at Pennsylvania State University, a research programmer at the IBM T.J. Watson Research Center, and a research consultant on the Space Station Freedom Project at the NASA Ames Research Center. Dr. Feng is a member of the IEEE and the ACM. 


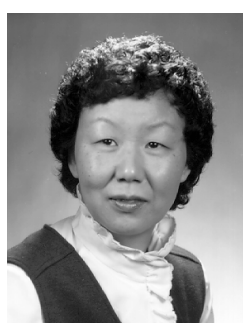

Jane W.-S. Liu received her BSEE degree in 1959 from the Cleveland State University, Ohio. She received her MS and ScD degrees in 1966 and 1968, respectively, from the Massachusetts Institute of Technology. She is currently a professor of computer science at the University of Illinois at Urbana-Champaign. Before joining the University of Illinois in 1973, she worked as an electronics engineer for the U.S. Department of Transportation, the Mitre Corporation, and the Radio Corporation of America. Her research interests are in the areas of real-time systems, distributed systems, and networks. She served as chair of the IEEE Computer Society Technical Committee on Data Base Engineering in 1981 and 1982, and chair of the Technical Committee on Distributed Processing in 1989 and 1990. She is the editor-in-chief of the IEEE Transactions on Computers and an associate editor of the International Real-Time Systems Journal. She is a fellow of the IEEE and a member of the ACM. 